\title{
An Efficient Healing and Hole Detection in Mobile Wireless Sensor Network Based on Distributed and Localized Algorithms
}

\author{
T. Suresh ${ }^{1}$, C. Anbuananth ${ }^{2}$ and G. Prabakaran ${ }^{3}$ \\ ${ }^{1,2 \& 3}$ Assistant Professor, Department of Computer Science and Engineering, \\ Annamalai Unviersity, Chidambaram, Tamil Nadu, India \\ E-Mail: sureshaucse@gmail.com, anbu_ananth2006@yahoo.com,gpaucse@yahoo.com
}

\begin{abstract}
The emerging trends of wireless sensor network (WSN) are estimated to afford a wide range of applications, such as battlefield surveillance, environmental monitoring, and smart spaces and so on. The coverage problem is an essential concern in the WSN. In this paper seeks to address the problem of hole detection and healing in mobile WSNs. This paper discuss the main drawbacks of existing solutions and identify four key elements that are critical for ensuring effective coverage in mobile WSNs: (i) Identifying the boundary of Region of Interest, (ii) Finding the coverage holes and estimating their characteristics, (iii) Identifying the best target locations to relocate mobile nodes to repair holes, and (iv) Dispatching mobile nodes to the target locations while minimizing the moving and messaging cost. This paper proposes a lightweight and comprehensive solution called Holes detection and healing, that addresses all of the aforementioned aspects. HEAL is a distributed and localized algorithm that operates in two distinct phases. First, a collaborative mechanism, called Distributed Hole Detection (DHD), is proposed to identify the boundary nodes and discover holes. Second, this paper presents a virtual forcebased hole healing algorithm. Unlike existing algorithms, HEAL algorithm relocates only the adequate nodes within the shortest times with the lowest cost. Simulation results shows that HEAL provide a cost-effective and an accurate solution. Keywords: WSN, Hole Detection, Healing Process
\end{abstract}

\section{INTRODUCTION}

Modern progresses in micro-electro-mechanical systems, embedded processors, and wireless communications have led to the emergence of Wireless Sensor Networks (WSNs), which consist of a large number of sensing devices each capable of sensing, processing and transmitting environmental information.

A significant dispute in WSNs is the coverage problem. The coverage problem [1] is heavily dependent on the coverage model of individual sensor and the locations of the deployed sensor nodes. Sensor coverage method can be deliberated as a quantity of the quality of service (QoS) of sensor's sensing task and is subject to a wide range of explanations due to a huge range of sensors and applications. In the Existing works, a commonly used sensor coverage model where a sensor can cover a disk placed at itself with a radius equal to a fixed sensing range. Network sensing coverage on the other hand can be deliberated as a shared measure of the QoS provided by sensor nodes at diverse geographical locations. In many cases, we may interpret the coverage concept as a non-negative mapping between the space points and the sensor nodes. WSN can be either systematically located or unsystematically located in a sensor field. In a systematic, sensor can be applied a small to medium network. When the network size is large or the sensor field is remote and hostile, unsystematically sensor can be used. To promise whole coverage in single unsystematic located, it is regularly assumed that the number of distributed sensors is more than that required by the critical sensor density. Mobile sensor nodes are armed with locomotive stages and can move everywhere after initial deployment.

The remaining of the paper is organized as follows the Section 2 describes the various related works and methods. The Section 3 explains the methodology adopted and the estimation procedures for Hole detection and Hole healing process. The Section 4 consists of the experiments and results conducted and finally the Section 5 concludes the paper.

\section{RELATED WORKS}

The main aim of this work is to provide a cost-effective and an accurate solution for hole detection and healing in mobile WSNs. In [1], Ghrist et al., proposes a new technique for detecting holes in coverage by means of homology. This technique is a completion of network communication graphs of two types of complexes: the nerve complex and the Rips complex. The previous gives evidence about coverage intersection of singular sensor nodes, and is very tough to compute. The later captures it is easy to compute but does not in itself return coverage data.

Fekete et al., [2] developed neighborhood-based topology recognition in sensor networks. It presented a coordinatefree method to identify boundaries in WSNs. They assume an unchanging node spreading in non-hole areas. It describes algorithmic approaches for determining the structure of boundary nodes of the region, and the topology of the region. It also develops methods for determining the outside boundary, the distance to the closest boundary for each point and the geometric thickness of the network.

Fang et al., [3] published locating and bypassing holes in sensor networks. Bound-Hole algorithm using the right- 
hand rule is proposed to identify nodes on the boundary of geometric holes. The Bound-Hole algorithm, being less dependent on location information and more tolerant of measurement inaccuracy, is a better choice for practical implementation.

In Wang et al., [4] represents the boundary recognition in sensor networks by topological method. It developed a practical distributed algorithm for boundary detection in sensor networks, using only the communication graph, and not making unrealistic assumptions. The boundary detection algorithm is motivated by an observation that holes in a sensor field create irregularities in hop count distances. This algorithm then focuses on finding the inner and outer boundaries of the network, which, with the cut nodes put back, will give the correct boundary cycles.

Li et al., [5] proposes strictly localized sensor selfdeployment for optimal focused coverage. It represents to deploy sensors in polygon layers over a locally computable equilateral triangle tessellation (TT) and introduce two types of deployment polygon, H-polygon and C-polygon. In this work, author introduces two algorithms. The two algorithms both yield a connected network of with hole-free coverage.

Wang et al., [6] have published a distributed selfdeployment protocols for mobile sensors. After determining a coverage hole, then we calculate the target positions of the sensors where they should move. Voronoi diagrams are used to discover the coverage holes and design three movement-assisted sensor deployment protocols such as Vector based, Voronoi based and Minimax based on the principle of moving sensors from densely deployed areas to sparsely deployed areas. The sensor deployment protocol runs iteratively until it terminates or reaches the specified maximum round.

Ganeriwal et al., [7] developed self-aware actuation for fault repair in sensor networks. It propose an algorithm referred to as Coverage Fidelity maintenance algorithm (CoFi) that uses mobility as an adaptive actuation facility for automated deployment repair of the network with the sole objective of salvaging the lost coverage in the network. It utilizes the mobility of nodes to set up a virtual flow of energy from one part of the system to another, creating an ad-hoc self-sustainable system that can last until the whole system become energy constrained rather than losing utility when only a few small regions of the system have been drained. Co-Fi can be seen as an extreme case of collaboration among the sensor nodes where a sensor node may sacrifice its existing role and expend energy on moving, to bring about a potential performance enhancement of the network as a whole [8].

\section{METHODOLOGY}

The major work of this paper is to design and evaluation of HEAL, that can effectively estimate and enhance the area coverage in a mobile WSNs. This work proposes a distributed virtual forces-based local healing approach based on the Hole Healing Area (HHA), in which the forces will be effective. This allows a local healing where only the nodes located at an appropriate distance from the hole will be involved in the healing process. The main advantage of this work is to provide a cost-effective and an accurate solution for hole detection and healing in mobile WSNs.

\section{A. Energy Efficient Hole Healing Process}

The arrangements of Hole healing process as shown in Fig 1. A border node with residual energy below a critical threshold may form new coverage-hole while healing the current coverage-hole. These nodes are known as hazardous border node. These nodes might generate coverage hole soon after their residual energy exhaustion. To solve this issue, the hole- manager (HM) broadcast messages requesting the energy level of all the boundary nodes. HM relocates these hazardous border nodes along with the hazardous nodes to prevent further formation of coverage hole in that particular region. This technique is more effective as it prevents the coverage-hole that is to be formed in the near future.

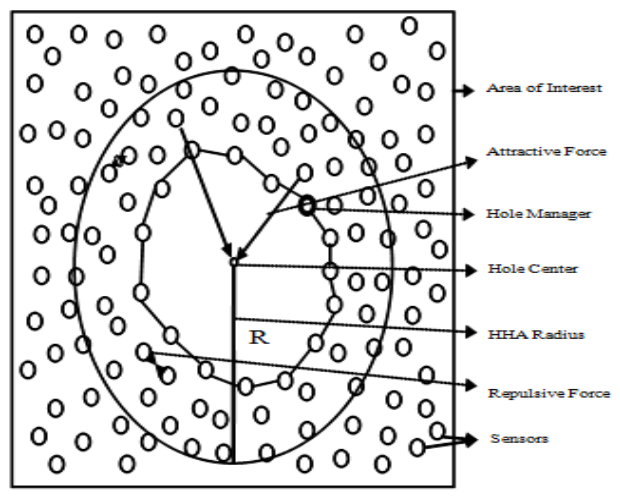

Fig.1 Architecture of Hole healing process

B. Implementation: The need for Hole detection and Hole healing process as shown in Fig. 2.

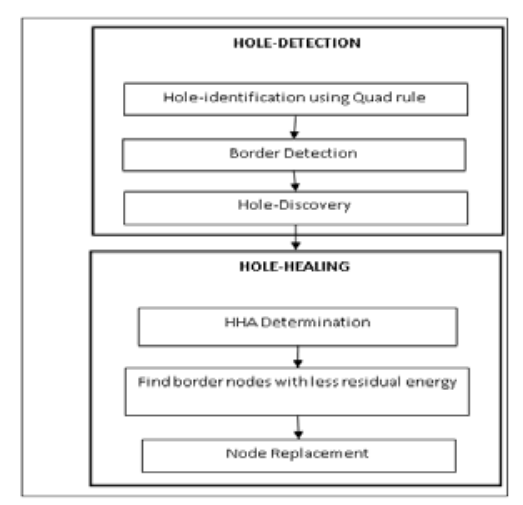

Fig.2 Block diagram of Hole detection and Hole healing process

\section{Hole-Identification}

The TENT rule specifies that a node is not a stuck node when there is no angle spanned by a pair of its angularly 
adjacent neighbors greater than $2 \pi / 3\left[120^{\circ}\right]$. While adapt this technique to conceive the distributed hole detection algorithm, it is unable to heal the hole completely as it checks for neighbor within the angle $120^{\circ}$. Quadrant based hole identification process is added in which each node checks for neighbors within $90^{\circ}$ instead of $120^{\circ}$. Each node divides the region into 4 quadrants by keeping itself as middle and checks for neighbors in each section. If a node is having at least single neighbor in every quadrant it is considered as stuck node. Quadrant based neighbor checking simplifies the stuck node identification process using TENT rule.

\section{Border Detection}

The network boundary nodes execute the Quad rule and, as a result, they detect that they are stuck nodes, which will launch the Hole discovery and the healing processes even if these nodes are actually not stuck nodes. To avoid the holediscovery we need to carry out the network boundary-node identification.

To discover the network boundary in a distributed way, we follow the following steps:

a. Each node of the network executes the Quad rule.

b. Each stuck node launches DHD to identify the nodes that surround the hole.

c. In the HD packet, we define four Boolean variables to identify the network boundary $X_{\max }, Y_{\max }, X_{\min }, Y_{\min }$. Each stuck node, which receives a HD packet, compares the coordinates $\mathrm{X}_{\max }, \mathrm{Y}_{\max }, \mathrm{X}_{\min }, \mathrm{Ymin}$ defined in the packet with its coordinates. Any one of these values related to all its neighbors, it sets the corresponding Boolean variable to 1 .

d. At the end of this procedure, the largest hole that defines the network boundary will be defined by the coordinates $X_{\max }, Y_{\max }, X_{\min }, Y_{\min }$.

e. Cancel the healing process that will be launched by the HM node.

\section{Hole-Discovery}

The goal of this phase is to find the boundary of the hole.A stuck node $b_{i}$ creates a new Hole-Discovery (HD) packet, marked with its ID, whose task is to collect location information of Hole boundary nodes, and forwards it to the next boundary node $b_{i+1}$. Node $b_{i+1}$ inserts its location information into the received HD packet and forwards it to the node $b_{i+2}$. This procedure is repetitive until the HD packet has covered around the hole and finally been received by the creator node $b_{i}$. Node bi extracts from the received HD packet the locations of the boundary nodes $\left\{b_{0}\right.$, $\left.\mathrm{b}_{1}, \ldots \ldots, \mathrm{b}_{\mathrm{n}}\right\}$. It selects two nodes $\mathrm{b}_{\mathrm{n}}$ and $\mathrm{b}_{\mathrm{m}}$ then we calculate distance of two nodes using the formula.

Distance $\left(b_{m}, b_{n}\right)=$ Max \{Distance $\left(b_{j}, b_{k}\right) / b_{j}, b_{k} \square b_{0}, b_{1}$, $\left.\left.\ldots . ., b_{n}\right\}\right\}$.Then, it calculates the Hole center, which is the mid-point $v$ of segment $b_{m} a n d b_{n}$.

$$
\begin{aligned}
& \mathrm{x}_{\mathrm{v}}=\left(\mathrm{x}_{\mathrm{km}}+\mathrm{x}_{\mathrm{kn}}\right) / 2 \\
& \mathrm{y}_{\mathrm{x}}=\left(\mathrm{y}_{\mathrm{km}}+\mathrm{y}_{\mathrm{kp}}\right) / 2
\end{aligned}
$$

Each stuck node sends a HD packet without any coordination among stuck nodes. Thus, there will be redundancy in the discovery process. This will generate unnecessary traffic and more packet collisions; the situation may become worse especially for large holes. To avoid this problem, a mechanism is used to prevent redundancy in the discovery process. The basic idea is to remove redundant HD packets as soon as possible. The criterion for judging whether a HD packet is redundant at each node, if a HoleID carried by a packet already passed, the packet will be considered redundant and it will be deleted. At the end of this step the node that has the smallest Hole-ID removes the HD packet and names itself as 'Hole Manager (HM): it will be responsible for the hole-healing announcement.

\section{Hole-Healing Area Determination}

This work exploits the nodes locomotion facilities to heal detected holes. Proposed relocation algorithm is completely distributed and it is based on the concept of virtual forces. To heal the discovered hole, it defines an attractive force that acts from the hole-center and attracts the nodes towards this center. Similarly, a repulsive force is defined among nodes to minimize the overlapping in between the nodes. It defines the Hole Healing Area (HHA).

\section{i. HHA Determination}

The HHA constitutes the basic idea of our algorithm; the determination of this area will determine the number of nodes that must be relocated to ensure a local repair of the hole. After the identification of the hole, the HM node calculates the middle and the size of the hole. Approximate the hole by a circle whose radius is the longest distance between two hole-boundary nodes. To determine the HHA, we need the radius of the circle. To find the appropriate radius, using the formula then we calculated.

$$
\mathrm{R}_{\mathrm{S}}=\mathrm{r} *\left(1+\mathrm{Q} \mathrm{\textrm {II }} \mathrm{R}_{\mathrm{S}}^{+}\right.
$$

Where ' $r$ ' is the hole radius. ' $\square$ s a positive constant, which depends on the nodes density and the sensing range $R_{S}$. For 目 0 , we start with a radius equals to the estimated hole radius $r$ due to the fact that this area may contain a sufficient nodes to heal the hole.The area defined by this circle is equal to ry. The number of nodes necessary to cover the area HHA-0 is equal to:

$$
\begin{aligned}
& \pi \mathrm{r}^{2} \mathrm{r}^{2} \\
& \overline{\pi \mathrm{RS}_{\mathrm{S}}{ }^{2} \mathrm{R}_{\mathrm{S}}{ }^{2}}-
\end{aligned}
$$

The HM node estimates the number of nodes existent in the HHA-0. It solicits its direct neighbors to calculate the number of their neighbors in this area. It sends a HHA Determination packet containing information about the hole. This communication is done over the GG to reduce the number of exchanged messages. If the number of nodes is less than the required number of the hole, the movement of these nodes will create new holes. To escape this state the 
HM node starts a new round by increasing ' $\square$ it repeats this process until it finds a sufficient number of nodes to recover the hole.

\section{E. Node Relocation}

After determining the HHA, the HM informs the nodes involved in the healing process. Nodes that receive forces from the hole-center move towards it. This section describes the concept of virtual forces used within the proposed HEAL algorithm.

\section{i. Attractive Force}

The Hole center ' $v$ ' applies an attractive force on every node within the HHA and located at a distance greater than $\mathrm{d}^{\text {th }}$ from $\mathrm{v}$. A node ' $\mathrm{p}$ ' within the HHA receives an attractive force $\mathrm{F}_{\mathrm{a}}(\mathrm{p}, \mathrm{v})$ form $\mathrm{v}$ given by:

$$
\begin{aligned}
& \begin{array}{l}
-\mathrm{k}_{\mathrm{A}}(\mathrm{I}) * \mathrm{e}^{(\mathrm{r} r}\left\{\frac{\mathrm{d}(\mathrm{p}, \mathrm{v}) \mathrm{u}_{\mathrm{A}}, \mathrm{d}(\mathrm{p}, \mathrm{v})>\mathrm{d}_{\mathrm{th}}^{\mathrm{a}}}{\mathrm{F}_{\mathrm{a}}(\mathrm{p}, \mathrm{v})=\mathrm{d}(\mathrm{p}, \mathrm{v}) \mathrm{1}_{\mathrm{a}}}\right.
\end{array} \\
& 0, \quad \mathrm{~d}(\mathrm{p}, \mathrm{v}) \leqslant \mathrm{d}^{\mathrm{a}} \mathrm{th}_{\mathrm{t}}(4)
\end{aligned}
$$

$\mathrm{u}_{\mathrm{A}}$ is the unit vector oriented from the point $\mathrm{p}$ to the Hole center v, d(p, v) the Euclidean distance between the node $\mathrm{p}$ and the hole center located at $\mathrm{v}(\mathrm{x}, \mathrm{y}) . \mathrm{l}_{\mathrm{a}}$ is a distance coefficient, $\mathrm{k}_{\mathrm{A}}$ is a coefficient that defines the intensity of the attractive force and $r$ is the hole radius.

The exponential factor enables us to control the movement of nodes in the HHA, so that the nodes closest to the holecenter will move longer distance than those on the boundary to prevent the creation of new holes during the healing process. The formulation of the exponential factor is a heuristic that we have proposed and that is based on the logic of the nodes movement, where the distance is inversely proportional to the force.

\section{ii. Repulsive Force}

To minimize overlapped coverage, a repulsive force exists among nodes within the range $0<\mathrm{F}_{\mathrm{r}}<\mathrm{d}_{\text {th }}^{\mathrm{r}}$ where $\mathrm{F}_{\mathrm{r}}$ denotes repulsive force. If the distance between two nodes $\mathrm{p}$ and $\mathrm{q}$ is less than $d_{\text {th }}^{r}$ then $F_{r}(p, q)$ will separate those nodes to minimize the overlapped coverage. $F_{r}(p, q)$ increases as the distance $d(p, v)$ decreases and is given by:

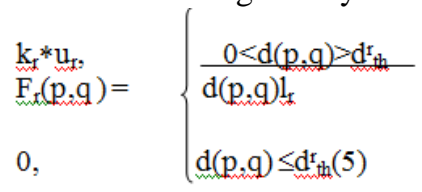

Where $u_{r}$ is the unit vector oriented from node $q$ to $p, k_{r}$ is a factor that defines the intensity of the repulsive force and $\mathrm{l}_{\mathrm{r}}$ is a distance coefficient such as $l_{r}>l_{a}$.

\section{iii. Movement Equation}

The final position of a node $\mathrm{p}$ is dictated by the resultant force of the composition of all repulsive forces that sustained the node from its Neighbors Set and the attractive force applied by the hole-center.
This force is $F_{p}$.

$$
\mathrm{F}_{\mathrm{p}}=\mathrm{F}_{\mathrm{a}}(\mathrm{p}, \mathrm{v})+\mathrm{F}_{\mathrm{r}}(\mathrm{p}, \mathrm{q})
$$

If $F_{p}=0$, then the node $p$ remains in its original position. Otherwise, $\mathrm{p}$ moves one time step in the direction imposed by $F_{p}$. The final position of $p$ is given by:

$$
\underset{\left\|\mathrm{F}_{p}\right\|}{\stackrel{\mathrm{F}_{p}}{\left.\mathrm{P}_{p}(t+\Delta t)\right]=}} * \mathrm{~V}+\mathrm{P}_{\mathrm{p}}(\mathrm{t})
$$

Where $\mathrm{V}$ is the node velocity and $\mathrm{P}_{\mathrm{p}}(\mathrm{t})$ is its position at instant $t$. The proposed HEAL can be extended by adding a new notion as follows: A border node with residual energy below a critical threshold may form new coverage-hole while healing the current coverage-hole. These nodes are known as hazardous border node. These nodes might generate coverage hole soon after their residual energy exhaustion. To solve this issue, the HM broadcast messages requesting the energy level of all the boundary nodes. HM relocates these hazardous border nodes along with the hazardous nodes to prevent further formation of coverage hole in that particular region. This technique is more effective as it prevents the coverage-hole that is to be formed in the near future.

\section{SIMULATION AND PERFORMANCE EVALUATIONS}

An extensive simulation model having scenario of $n$ mobile nodes and n UDP/TCP connections is used to study interlayer interactions and their performance implications. The other parameters used in this model are given in Table I.

TABLE I SIMULATION PARAMETERS

\begin{tabular}{|l|c|}
\hline Software for simulation & Network simulator 2 \\
\hline Channel & Wireless \\
\hline Simulation runs time & 50 seconds \\
\hline Area in which nodes move & 200 X 200 \\
\hline Packet size & 1024 bytes \\
\hline Speed & $1 \mathrm{~m} / \mathrm{s}$ to $10 \mathrm{~m} / \mathrm{s}$ \\
\hline Routing Protocol & AODV \\
\hline Propagation model & TwoRayGround \\
\hline Network Interface Type & Wireless Physical \\
\hline Queue Type & Drop Tail \\
\hline IFQ-Length & 50 Packets \\
\hline MAC Type & Mac/802.11 \\
\hline Antenna Type & Omni Antenna \\
\hline
\end{tabular}

The performance differentials are analyzed using number of movements and coverage percentage.

\section{A. Performance Metrics}

1. Coverage Percentage: The percentage of area that is covered at the end of the coverage-Hole healing phase

2. Number of Movements: It is the number of movements made by a node to heal the coverage-Hole. 


\section{B. Experimental Results}

The Experimental purpose, we have implemented NS-2 simulation. In Fig 3 shows Initial deployment of nodes, this is done by varying size and position of the hole created.

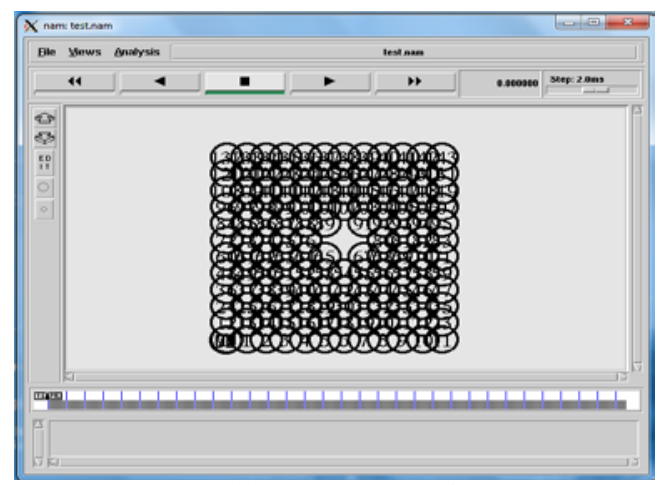

Fig. 3 Initial Deployment of nodes

A node which is not able to communicate at least any one direction in the network then it is known as stuck nodes. Nodes changed to tomato color are stuck nodes as shown in Fig 4.

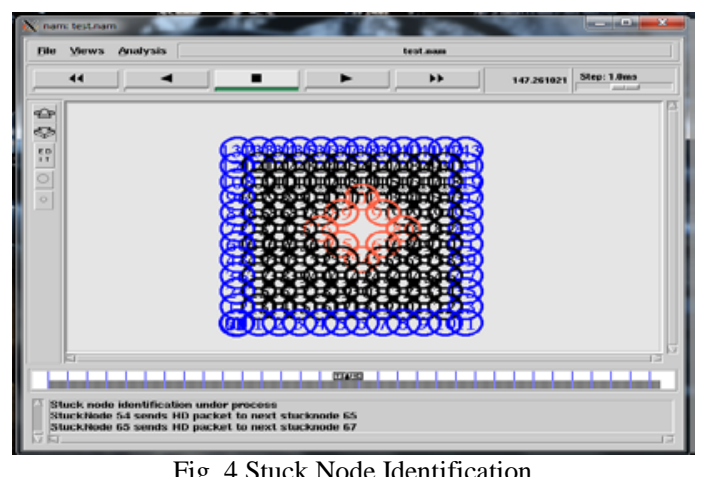

In Fig 5 represents the completion of Hole Healing process after which packet exchange takes place to check the presence of holes and boundary. Finally, node area and Hole healing area are calculated by evaluating the performance metrics.

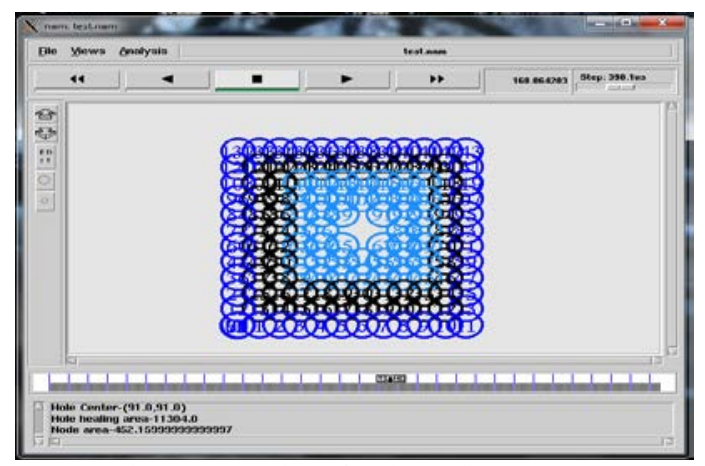

Fig. 5 Completion of Hole Healing process

\section{CONCLUSION}

This paper has detected and implemented a lightweight and comprehensive two-phase protocol such as HEAL, for ensuring area coverage employing a mobile WSN. The protocol uses a distributed hole-detection algorithm (DHD) to detect holes in the network. Compared to the existing schemes, DHD deals with holes of various forms and sizes despite the nodes distribution and density. By exploiting the virtual forces concept, our approach relocates only the adequate nodes within the shortest time and at the lowest cost. Quad rule based hole stuck node identification process ensures the precise detection of hole. During the performance evaluation, it is examined HEAL, using different criteria and showed that it detects and heals the holes despite their number or size with less mobility in various situations. The evaluation results demonstrate that better performance in terms of cost-effective and an accurate solution for hole detection and healing in mobile WSNs.In future it may be extended to apply for MANET applications.

\section{REFERENCES}

[1] R. Ghrist, and A. Muhammad, "Coverage and Hole-Detection in Sensor Networks via Homology”, in the Proc. IPSN'05, pp. 254-260

[2] S.P. Fekete, A. Kroller, D. Pfisterer, S. Fischer, and C. Buschmann, "Neighborhood-based topology recognition in sensor networks", in Proc. Algo-sensors, Springer LNCS,Vol. 3121, pp. 123-136, 2004

[3] Q. Fang, Gao and Guibas, "Locating and Bypassing holes in Sensor Networks” Mobile Networks and Applications, Vol.11, No.2 pp. 187200, 2006.

[4] Y. Wang, J. Gao, and S.B. Mitchell, "Boundary recognition in sensor networks by topological methods”, MobiCom, ACM, pp. 122-133, 2006

[5] X. Li, H. Frey and N. Santoro "Strictly Localized Sensor SelfDeployment for Optimal Focused Coverage” IEEE Transactionson Mobile Computing, Vol. 10, No.11, pp. 1520-1533, 2011.

[6] Wang and La Porta, "Movement-Assisted Sensor Deployment", IEEE Transactions on Mobile Computing, Vol. 5, No.6, pp. 640-652, 2006.

[7] S. Ganeriwal, A. Kansal, and M.B. Srivastav, "Self-aware actuation for fault repair in sensor networks” IEEE International Conference on Roboticsand Automation (ICRA'04), pp. 5244-5249, 2004.

[8] K. Stephen, and E. Anna devi, “A Review on Hole detection and Healing in Wireless sensor Network" International Journal of Engineering Research and General science, Vol. 3, No. 1, pp. 10911094, 2015. 